Dynamical Entropy of Generalized Quantum Markov Chains

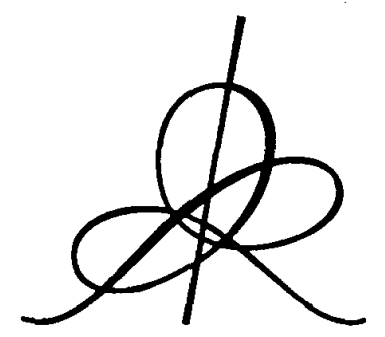

Institut des Hautes Etudes Scientifiques 35 , route de Chartres

91440 - Bures-sur-Yvette (France)

Novembre 1993

IHES $/ \mathrm{P} / 93 / 60$ 



\title{
Dynamical Entropy of Generalized Quantum Markov Chains
}

\author{
Yong Moon Park ${ }^{1, *, * *}$
}

${ }^{1}$ Institut des Hautes Etudes Scientifiques, F-91440 Bures-sur-Yvette, France

\begin{abstract}
We compute the dynamical entropy in the sense of Connes, Narnhofer and Thirring of shift automorphism of generalized quantum Markov chains as defined by Accardi and Frigerio. For any generalized quantum Markor chain defined via a finite set of conditional density amplitudes we show that the dynamical entropy is equal to the mean entropy.
\end{abstract}

* Permanent address : Department of Mathematics, Yonsei University, Seoul 120-749, Korea

** Research supported in part by the Basic Science Research Program, Korean Ministry of Education, 1993-1994. 


\section{Introduction}

In their paper [5] Connes, Narnhofer and Thirring (CNT) extended the notion of dynamical entropy of classical dynamical systems introduced by Kolmogorov and Sinai $[7,14]$ to the case of automorphisms of $C^{*}$-algebras invariant with respect to a given state. As in the classical ergodic theory [18], the concept of the CNT entropy should be mathematically useful to find a classification of quantum chaotic systems. In this respect it should be important to develop the method which enable to compute the entropy for quantum systems. There have been many results on entropic computations ([8-12, 15-17] and references therein).

In this paper we compute the CNT entropy of shift automorphisms of generalized quantum Markov chains (GQMC) as defined by Accardi and Frigerio [1]. For any GQMC defined via a finite set of conditional density amplitudes we show that the dynamical (CNT) entropy is equal to the mean entropy.

It should be mentioned that Besson [3] computed the Connes-Størmer (CS) entropy [6] of shift automorphism of quantum Markor chains (QMC). Later on the result was generalized by Quasthoff [13]. See also chapter 11 of Ref. [10] for additional informations. But, as mentioned in Ref. [1], one has to deal with the large class of GQMC in order to account the nearest neighbours potentials of interest for quantum statistical mechanics. Also it has been shown in [1] that any QMC belongs to the class we consider and so our result can be viewed as a non-trivial extension of that in [3]. On the other hand we are only able to deal with GQMC defined via conditional density amplitudes. Thus it must be desirable to find the method which is applicable to GQMC defined via a transition expectation directly.

We organize the paper as follows: In Section 2 we recall the definitions of generalized quantum Markov chains [1,2] and then describe the class which we consider in this paper. In Section 3 we review the dynamical entropy of $C^{*}$-algebra and then state our main theorem. In Section 4 we procluce the proof of the main theorem. 


\section{Generalized Quantum Markov Chain}

Let us recall the definition of generalized Quantum Markov chains [1,2]. Let $M_{0} \subset$ $M_{d}(\mathbf{C}), d>1$, be a fixed subalgebra of $M_{d}(\mathbf{C})$ (the $d \times d$ complex matrices), and $\mathfrak{A}$ be the $C^{*}$-algebra $\mathfrak{A}=\otimes_{\mathbf{Z}} M_{0}$. Let $J_{n}$ be the canonical injection of $M_{0}$ into the $n$-th factor of $\mathfrak{A}$. For $I \subset \mathbf{Z}$ we denote by $\mathfrak{L}_{I}$ the algebra generated by $\left\{J_{n}\left(M_{0}\right): n \in I\right\}$. In the above notation we have

$$
\mathfrak{A}=\left(\bigcup_{I \subset \mathbf{Z}} \mathfrak{A}_{1}\right)^{-}, \mathfrak{A}_{I}=\otimes_{n \in I} J_{n}\left(M_{0}\right)
$$

where the bar denotes the norm closure.

The basic ingredients in the construction of a stationary (generalized) quantum Markov chain in the sense of Accardi and Frigerio [1] consist of a completely positive unital map (c.p.u.map) E, called a transition expectation :

$$
\begin{gathered}
E: M_{\mathbf{0}} \odot M_{0} \rightarrow M_{0} \\
E(\mathbf{1} \odot \mathbf{1})=\mathbf{1}
\end{gathered}
$$

and a state $\phi_{0}$ on $M_{0}$, satisfying the following condition :

$$
\phi_{0}(E(1 \Theta x))=\phi_{0}(x), x \in M_{0}
$$

For any $\Lambda=[i, k] \equiv\{i, i+1, \cdots, k\} \subset \mathbf{Z}$ and $x_{j} \in M_{0}, i \leq j \leq k$, one then defines

$$
\begin{aligned}
& \omega_{\Lambda}\left(x_{i} \otimes x_{i+1} \oslash \cdots \otimes x_{k}\right) \\
& \quad=\phi_{0}\left(E\left(x_{i} \otimes E\left(x_{i+1} \oslash E\left(x_{i+2} \bigcirc \cdots \odot E\left(x_{k} \bigcirc 1\right) \cdots\right)\right)\right)\right) .
\end{aligned}
$$

Notice that the family of local states $\left\{\omega_{\Lambda}: \Lambda \subset Z\right\}$ satisfies a compatible condition by (2.2) and (2.3), and the state $\omega$ defined by $\omega_{\Lambda}, \Lambda \subset \mathrm{Z}$, is stationary.

Definition $2.1[1,2]$. (a) Let $E$ be a transition expectation satisfying (2.2) and let $\phi_{0}$ be a state on $M_{0}$ satisfying (2.3). Then $\left(\phi_{0}, E\right)$ is called a Markov pair.

(b) The state $\omega$ on $\mathfrak{A}$ defined by the sequence $\left\{\omega_{[-n, n]}\right\}_{n \geq 1}$ is called a stationary generalized quantum Markov state. The dynamical system $(2, \alpha, \omega)$ is called a generalized quantum Markov chain, where $\alpha$ is the shift map on $\mathfrak{A}, \alpha\left(J_{n}(a)\right)=J_{n+1}(a)$.

Notice that by (2.3) the state $\omega$ is invariant with respect to the shift $\alpha$. 
Remark 2.2. If the transition expectation satisfies the additional condition :

$$
E(x \otimes y)=E(x \otimes E(y \otimes 1)), x, y \in M_{0}, \bmod .\left\{\phi_{0}, E\right\},
$$

then the corresponding dynamical system is called a Quantum Markov Chain [1].

In this paper we consider the generalized quantum Markov chains defined by a finite set of conditional density amplitudes $[1,2]$. We first consider the generalized quantum markov chain defined via a single conditional density amplitude. Let $\mathrm{Tr}$ be the trace on $M_{0}$ which takes the value 1 at each minimal projection, and let $\widetilde{\operatorname{Tr}}$ be the trace on $M_{0} \bigcirc M_{0}$. Denote by $\widetilde{\operatorname{Tr}}^{(i)}, i=1,2$, the partial traces defined by

$$
\widetilde{\operatorname{Tr}}^{(1)}(a \odot b)=\operatorname{Tr}(a) b \quad \widetilde{\operatorname{Tr}}^{(2)}(a \bigcirc b)=\operatorname{Tr}(b) a
$$

Let $W_{0} \in M_{0}$ be a density matrix in $M_{0}\left(W_{0} \geq 0\right.$ and $\left.\operatorname{Tr}\left(W_{0}\right)=1\right)$ and let $K \in M_{0} \odot M_{0}$ be an operator satisfying

$$
\widetilde{\mathrm{Tr}}^{(2)}\left(I \Lambda^{*}\right)=1, \quad \widetilde{\mathrm{Tr}}^{(1)}\left(I^{-*}\left(W_{0} \odot 1\right) \Pi^{-}\right)=W_{0}
$$

A positive operator $\Lambda \in M_{0} \odot M_{0}$ satisfying the above is called a conditional density amplitude $[1,2]$. For given conditional density amplitude $K$, one can define a transition expectation $E$ by

$$
E(x)=\widetilde{\operatorname{Tr}}^{(2)}\left(\Lambda_{x} x \Lambda^{-*}\right), \quad x \in M_{0} \odot M_{0}
$$

Let $\phi_{0}$ be the state on $M_{0}$ defined by the density matrix $W_{0}$. Then it follows from (2.5) and (2.6) that $\left(\phi_{0}, E\right)$ is a Markov pair generated by the pair $\left(W_{0}, K\right)$. For a conditional density amplityde $K$, and for $i, k, n \in \mathrm{Z}$. let

$$
\begin{aligned}
I_{[n, n+1]} & =\left(J_{n} \odot J_{n+1}\right)\left(K^{r}\right), \\
K_{[i, k+1]} & =I_{[i, i+1]} I_{[i+1, i+2]}^{-} \cdots K_{[k, k+1]}, i \leq k
\end{aligned}
$$

We write that

$$
W_{[i, k+1]}=\Lambda_{[i, k+1]}^{*} J_{i}\left(W_{0}\right) I_{[i, k+1]}^{-}
$$

It then follows from (2.4) and (2.7) that

$$
\omega_{[i, k]}\left(x_{i} \otimes x_{i+1} \odot \cdots \odot x_{k}\right)=\operatorname{Tr}\left(W_{[i, k+1]}\left(x_{i} \odot x_{i+1} \odot \cdots \odot x_{k} \odot 1\right)\right),
$$


where $\operatorname{Tr}$ is the trace on $\mathfrak{A}_{[i, k+1]}$.

The above construction of transition expectation can be generalized as follows : Let $\left\{K_{1}, K_{2}, \cdots, K_{l}\right\}$ be a finite subset of $M_{0} \otimes M_{0}$ satisfying

$$
\sum_{j=1}^{l} \widetilde{\operatorname{Tr}}^{(2)}\left(K_{j} K_{j}^{*}\right)=1, \quad \sum_{j=1}^{l} \widetilde{\operatorname{Tr}}^{(1)}\left(K_{j}^{*}\left(W_{0} \odot 1\right) K_{j}\right)=W_{0}
$$

where $W_{0} \in M_{0}$ is a density matrix. Then the most general transition expectation has the form [2]

$$
E(x)=\sum_{j=1}^{l} \widetilde{\operatorname{Tr}}^{(2)}\left(K_{j}^{*} x K_{j}\right), \quad x \in M_{0} \otimes M_{0}
$$

The set $\left\{K_{1}, K_{2}, \cdots, K_{l}\right\} \subset M_{0} \odot M_{0}$ satisfying the conditions in (2.11) will be called a set of conditional density amplitudes.

Next let us consider the mean entropy. For a given state $w$ on $\mathfrak{A}$ and finite $I \subset \mathbf{Z}$, let $S\left(\omega \mid \mathfrak{A}_{I}\right)$ be the entropy of the state $\omega$ on $\mathfrak{A}_{I}[5,10]$, where $\omega \mid \mathfrak{A}_{I}$ is the state on $\mathfrak{A}_{I}$ defined by the restriction of $\omega$ on $\mathfrak{A}_{I}$. If $\omega \mid \mathfrak{A}_{I}$ is given by normalized density matrix $\rho_{I}\left(\operatorname{Tr}\left(\rho_{I}\right)=1\right)$, then

$$
S\left(\omega \mid \mathscr{2}_{I}\right)=-\operatorname{Tr}\left(\rho_{I} \ln \rho_{I}\right) .
$$

By the subadditivity of $S\left(\omega \mid \mathfrak{A}_{1}\right)$ [4], the limit

$$
s(\omega)=\lim _{n \rightarrow \infty} \frac{1}{n+1} S\left(\omega \mid \mathfrak{A}_{[0, n]}\right)
$$

exists. The quantity $s(\omega)$ is called the mean entropy of the state $\omega$ on $\mathfrak{A}$. 


\section{Dynamical Entropy and Main Result}

Since $C^{*}$-algebra $\mathfrak{A}$ which we are dealing with is approximately finite $(A F)$, we review entropic results for AF-algebras. For the general definition of the CNT entropy and related results we refer to [5]. Let $(\mathfrak{A}, \alpha, \omega)$ be a $C^{*}$-dynamical system, where $\mathfrak{A}$ is a unital $C^{*}$ algebra of AF type, $\alpha$ is an automorphism on $\mathfrak{A}$ and $\omega$ is a state on $\mathfrak{A}$ which is invariant with respect to $\alpha$.

For a given state $\omega$ on $\mathfrak{A}$ and $k \in \mathbf{N}$, let $\left\{\omega_{i_{1} i_{2} \ldots i_{k}}: i_{j} \in \mathbf{N}, j=1, \cdots, k\right\}$ be a finite decomposition of the state $\omega$ :

$$
\omega=\sum_{i_{1}, i_{2}, \cdots, i_{k}} \omega_{i_{1} i_{2}} \cdots i_{k}
$$

Let $\eta$ be the function on $[0,1]$ defined by $\eta(x)=-x \ln x$ if $x>0$ and $\eta(0)=0$. We write

$$
\omega_{i_{l}}^{(l)}=\sum_{\substack{i_{1}, \cdots, i_{k}: \\ i_{l} \text { fixed }}} \omega_{i_{1} i_{2} \cdots i_{k}} \text { and } \hat{\omega}_{i_{l}}^{(l)}=\omega_{i_{l}}^{(l)} / \omega_{i_{l}}^{(l)}(\mathbf{1}) \text {. }
$$

For given finite dimensional subalgebras $\mathfrak{A}_{1}, \mathfrak{A}_{2}, \cdots, \mathfrak{A}_{k} \subset \mathfrak{A}$, put

$$
\begin{aligned}
& H_{\omega}\left(\mathfrak{A}_{1}, \mathfrak{A}_{2}, \cdots, \mathfrak{A}_{k}\right) \\
& =\sup _{\sum \omega_{i_{1}} \cdots i_{k}=\omega}\left[\sum_{i_{1}, \cdots, i_{k}} \eta\left(\omega_{i_{1} \cdots i_{k}}(\mathbf{1})\right)-\sum_{l=1}^{k} \sum_{i_{l}} \eta\left(\omega_{i_{l}}^{(l)}(\mathbf{1})\right)\right. \\
& \left.+\sum_{l=1}^{k} S\left(\omega \mid \mathfrak{A}_{l}\right)-\sum_{l=1}^{k} \sum_{i_{l}} \omega_{i_{l}}^{(l)}(\mathbf{1}) S\left(\hat{\omega}_{i_{l}}^{(l)} \mid \mathfrak{A}_{l}\right)\right],
\end{aligned}
$$

where the sup is taken over finite decompositions of $\omega$. For any finite dimensional subalgebra $\mathfrak{N} \subset \mathfrak{A}$, let

$$
h_{\omega, a}(\mathfrak{N})=\lim _{k \rightarrow \infty} \frac{1}{k} H_{\omega}\left(\mathfrak{N}, \alpha(\mathfrak{N}), \cdots, a^{k-1}(\mathfrak{N})\right) .
$$

Let $h_{\omega}(\alpha)$ be the dynamical entropy defined as in [5]. For an AF-algebra $\mathfrak{A}=$ $\left(\bigcup_{n=1}^{\infty} \mathfrak{A}_{n}\right)^{-}$with $1 \in \mathfrak{A}_{1} \subset \mathfrak{A}_{2} \cdots$, finite dimensional, it turned out that the equality

$$
h_{\omega}(\alpha)=\lim _{n} h_{\omega, \alpha}\left(\mathfrak{A}_{n}\right)
$$

holds [5, Corollary V.4]. This Liolmogorov-Sinai type theorem has been extended to the setting of quasi local algebras in quantum statistical mechanics [11]. 
For later use we collect some entropic results from [5].

Lemma 3.1 [5, Proposition III.6]. (a) If $\mathfrak{A}_{i}^{\prime} \subset \mathfrak{A}_{i}, i=1, \cdots, n$, then $H_{\omega}\left(\mathfrak{A}_{1}^{\prime}, \mathfrak{A}_{2}^{\prime}, \cdots, \mathfrak{A}_{n}^{\prime}\right)$ $\leq H_{\omega}\left(\mathfrak{A}_{1}, \mathfrak{A}_{2}, \cdots, \mathfrak{A}_{n}\right)$.

(b) $H_{\omega}\left(\mathfrak{A}_{1}, \mathfrak{A}_{2}, \cdots, \mathfrak{A}_{n}\right)$ depends only upon the set $\left\{\mathfrak{A}_{1}, \mathfrak{A}_{2}, \cdots, \mathfrak{A}_{n}\right\}=X$.

(c) The inequalities

$$
\max \left\{H_{\omega}(X), H_{\omega}(Y)\right\} \leq H_{\omega}(X \cup Y) \leq H_{\omega}(X)+H_{\omega}(Y)
$$

hold with the notation in (b).

The above results are consequences of general results in [5].

We now state our main result. Let $(\mathfrak{A}, \alpha, \omega)$ be a generalized quantum Markov chain introduced in Section 2, where $\alpha: \mathfrak{A} \rightarrow \mathfrak{A}$ is the shift automorphism.

Theorem 3.2. Let $W_{0} \in M_{0}$ and $\left\{K_{1}, K_{2}, \cdots, K_{l}\right\} \subset M_{0} \odot M_{0}$ be a density matrix and a finite set of conditional density amplitudes satisfying (2.11) and let $(\mathfrak{A}, \alpha, \omega)$ be the generalized quantum Markov chain constructed from $W_{0}$ and $\left\{K_{1}, K_{2}, \cdots, K_{l}\right\}$. Then the equality

$$
h_{\omega}(\alpha)=s(\omega)
$$

holds, where $s(\omega)$ is the mean entropy.

We will produce the proof of the above theorem in the next section. 


\section{Proof of Main Theorem}

We prove Theorem 3.2 in this section. Notice that by (3.4) the dynamical entropy of a generalized quantum Markov chain $(\mathfrak{A}, \alpha, \omega)$ is given by

$$
\begin{aligned}
h_{\omega}(\alpha) & =\lim _{n \rightarrow \infty} h_{\omega, \alpha}\left(\mathfrak{A}_{[-n, n]}\right) \\
& =\lim _{n \rightarrow \infty} h_{\omega, \alpha}\left(\mathfrak{A}_{[1, n]}\right) .
\end{aligned}
$$

Here we have used the fact that $h_{\omega, \alpha}\left(\mathfrak{A}_{[-n, n]}\right)=h_{\omega, \alpha}\left(\mathfrak{A}_{[1,2 n+1]}\right)$ which follows from the invariance of $\omega$ with respect to $\alpha$ and the expression in (3.2). The upper bound $\left(h_{\omega}(\alpha) \leq\right.$ $s(\omega))$ follows from Lemma $3.1,(3.3)$ and the fact that $H_{\omega}\left(\mathfrak{A}_{n}\right) \leq S\left(\omega \mid \mathfrak{R}_{n}\right)[5]$ :

$$
\begin{aligned}
H_{\omega}\left(\mathfrak{A}_{[1, n]}\right. & \left.\alpha\left(\mathfrak{A}_{[1, n]}\right), \cdots, \alpha^{k}\left(\mathfrak{A}_{[1, n]}\right)\right) \\
& \leq H_{\omega}\left(\mathfrak{2}_{[1, n+k]}\right) \\
& \leq S\left(\mathfrak{A}_{[1, n+k]}\right) .
\end{aligned}
$$

Dividing the above by $k+1$ and taking $k$ to infinity we get the upper bound.

Thus we need to get the lower bound $h_{\omega}(\alpha) \geq s(\omega)$. From Lemma 3.1 (c) it follows that

$$
\begin{aligned}
H_{\omega}\left(\mathfrak{A}_{[1, n]}, \alpha\left(\mathfrak{A}_{[1, n]}\right), \cdots, \alpha^{n k}\left(\mathfrak{A}_{[1, n]}\right)\right) \\
\quad \geq H_{\omega}\left(\mathfrak{A}_{[1, n]}, \mathfrak{A}_{[n+1,2 n]}, \cdots, \mathfrak{A}_{[n(k-1)+1, n k]}\right)
\end{aligned}
$$

In order to show the main idea of the proof more clearly, we first consider the case in which the transition expectation is defined by a single conditional density amplitude $K$ as in (2.7). To obtain the lower bound we have to choose an optimal clecomposition of the state $\omega$. For given $[l, m] \subset \mathrm{Z}$, let $\left\{x_{j}\right\} \subset \mathfrak{2}_{[l, m]}^{+}$be a finite decomposition of unity:

$$
\sum_{J} x_{J}=1, \quad x_{J} \geq 0, \quad x_{J} \in \mathfrak{A}_{[l, m]} .
$$

For $[l, m] \subset[i+1, k-1]$, define states on $\mathfrak{A}_{[i, k]}$ by

$$
\omega_{[i, k], J}(y) \equiv \operatorname{Tr}\left(\Lambda_{[i, k+1]}^{*} x_{J} J_{i}\left(W_{0}\right) \Lambda_{[i, k+1]}^{-} y\right), y \in \mathfrak{A}_{[i, k]}
$$


Notice that $x_{I}$ and $J_{i}\left(W_{0}\right)$ commute. It follows from $(2.9),(2.10)$ and (4.3) that

$$
\omega_{[i, k]}=\sum_{J} \omega_{[i, k], J}
$$

One may check that the family of states $\left\{\omega_{[i, k], J}: i \leq l, m \leq k\right\}$ is compatible. Let $\omega_{J}$ be the state on $\mathfrak{A}$ which is the extension of $\left\{\omega_{[i, k], J}\right\}$. Then we have

$$
\sum_{J} \omega_{J}=\omega
$$

Thus from a decomposition of unity $\left\{x_{J}\right\}$ we obtain a decomposition of the state $\omega$.

Next we choose a decomposition of unity explicitly. let $\left\{P_{1}, P_{2}, \cdots, P_{d^{\prime}}\right\} \subset M_{0}$ be a family of minimal projections in $M_{0}$. Recall the expression in (4.2). For fixed $k \geq 1$ and $n \geq 1$, let $\mathcal{I}$ be the multi-indices given by

$$
\mathcal{I}=\left\{J=\left(J_{1}, J_{2}, \cdots, J_{k}\right): J_{l} \in\left\{1,2, \cdots, d^{\prime}\right\}^{n}\right\}
$$

For each $J_{l}=\left(i_{1}, i_{2}, \cdots, i_{n}\right) \in\left\{1,2, \cdots, d^{\prime}\right\}^{n}$, put

$$
P_{J_{l}}=J_{n(l-1)+1}\left(P_{i_{1}}\right) \cdot J_{n(l-1)+2}\left(P_{i_{2}}\right) \ldots J_{n l}\left(P_{i_{n}}\right)
$$

and for given $J=\left(J_{1}, \cdots, J_{k}\right)$ we write

$$
P_{J}=P_{J_{1}} P_{J_{2}} \cdots P_{J_{k}}
$$

Then from the above definitions we have

$$
\sum_{J \in \mathcal{I}} P_{J}=1 \text { and } P_{J_{l}}=\sum_{\substack{J: \\ J_{l} \text { fixed }}} P_{J}
$$

Denote by $\left\{\omega_{J}\right\}_{J \in \mathcal{I}}$ the decomposition of the state obtained from the decomposition of unity $\left\{P_{J}\right\}_{J \in \mathcal{I}}$ by (4.3). From (4.3) and (4.7) it follows that from $J=\left(J_{1}, J_{2}, \cdots, J_{k}\right)$ and $l \in\{1,2, \cdots, k\}$

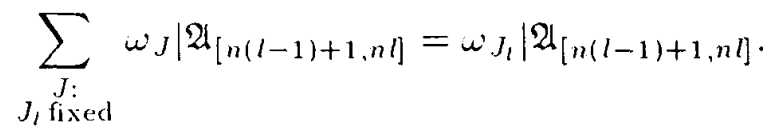


We now use (4.7) and the shift invariance of $\omega$ to obtain from (4.2) that

$$
\begin{aligned}
H_{\omega}\left(\mathfrak{A}_{[1, n]}, \mathfrak{A}_{[n+1,2 n]}, \cdots, \mathfrak{A}_{[n(k-1)+1, n k]}\right) \\
\quad \geq \sum_{J \in \mathcal{I}} \eta\left(\omega_{J}(\mathbf{1})\right)-k \sum_{I \in\left\{1, \cdots, d^{\prime}\right\}^{n}} \eta\left(\omega_{I}(\mathbf{1})\right) \\
+k S\left(\omega^{\prime} \mid \mathfrak{A}_{[1, n]}\right)-k \sum_{I \in\left\{1, \cdots, d^{\prime}\right\}^{n}} \widehat{\omega}_{I}(\mathbf{1}) S\left(\widehat{\omega}_{I} \mid \mathfrak{A}_{[1, n]}\right) .
\end{aligned}
$$

The main idea to obtain the lower bound is to show that $S\left(\widehat{\omega}_{I} \mid \mathfrak{A}_{[1, n]}\right) / n$ tends to zero as $n$ tends to infinity.

Proposition 4.1. There exists a constant $c$ independent of $n \in N$ and $I \in\left\{1,2, \cdots, d^{\prime}\right\}^{n}$ such that the bound

$$
S\left(\widehat{\omega}_{I} \mid \mathfrak{A}_{[1, n]}\right) \leq c
$$

hold uniformly in $n$ and $I$.

We postpone the proof of the abore result. Now Theorem 3.2 for $l=1$ is a consequence of Proposition 4.1.

Proof of Theorem 3.2 for $l=1$. Let $\mathfrak{B}_{0}$ be the abelian $C^{*}$-algebra generated by $\left\{P_{1}, P_{2}, \cdots, P_{d^{\prime}}\right\} \subset M_{0}$ and $\mathfrak{B}=\left(\odot \mathfrak{B}_{0}\right)^{\mathbf{Z}}$. As in Section 2, put

$$
\mathfrak{B}_{[l, m]}=\bigodot_{i=l}^{m} J_{i}\left(\mathfrak{B}_{0}\right)
$$

for any $[l, m] \subset \mathbf{Z}$. Define a state $\mu$ on $\mathfrak{B}$ by

$$
\mu\left(P_{J}\right)=\omega_{J}(\mathbf{1}), \quad P_{J} \in \mathfrak{B}_{[l, m]}
$$

where for $J \in\left\{1,2, \cdots, d^{\prime}\right\}^{[l, m]}, P_{J}=J_{l}\left(P_{i_{1}}\right) \cdots J_{m}\left(P_{i_{m}}\right)$. Then it follows that

$$
\sum_{J \in\left\{1,2, \cdots, d^{\prime}\right\}} \eta\left(\omega_{J}(\mathbf{1})=S_{\mu}\left(\mathfrak{B}_{[l, m]}\right)\right.
$$

where $S_{\mu}\left(\mathfrak{B}_{[l, m]}\right)$ is the classical entropy. Since the classical mean entropy exists, we have

$$
\lim _{n \rightarrow \infty} \lim _{k \rightarrow \infty} \frac{1}{k n}\left\{\sum_{J \in \mathcal{I}} \mu\left(\omega_{J}(J(\mathbf{1}))-k: \sum_{I \in\left\{1,2, \cdots, d^{\prime}\right\}^{n}} \eta\left(\omega_{I}(\mathbf{1})\right)\right\}=0\right.
$$


We now use (4.1), (4.2) and (4.8). We then divide both sides of (4.8) by $n k$ and take the limits. Then Theorem 4.2 follows from (4.9) and Proposition 4.1. This completes the proof of the main theorem for $l=1$.

Next we prove Proposition 4.1. We first need the following lemma :

Lemma 4.2. Let $\mathfrak{H}$ be a finite dimensional Hilbert space and let $\mathfrak{N}$ be a subalgebra of $\mathfrak{L}(\mathfrak{H})$. For given minimal projection $P \in \mathfrak{N}$ and $A \in \mathfrak{N}$, put

$$
Q=A P A^{*} / \operatorname{Tr}\left(A P A^{*}\right)
$$

Then $Q$ is a minimal projection in $\mathfrak{N}$.

Proof. Since $\mathfrak{N}$ is isomorphic to $\bigoplus_{j=1}^{k} M_{n_{j}}$, one may assume that any minimal projection is a rank one projection. We first show that $Q$ is a projection. Since

$$
Q^{2}=A P A^{*} A P A^{*} /\left(\operatorname{Tr}\left(A P A^{*}\right)\right)^{2}
$$

and since $P A^{*} A P=\lambda P$ by the minimality of $P$, where $\lambda=\operatorname{Tr}\left(P A^{*} A P\right)$, it follows that $Q^{2}=Q$. Since $A P A^{*}$ is a rank one operator, $Q$ is a minimal projection.

Proof of Proposition 4.1. Recall the definition of $\omega_{I}$ :

$$
\omega_{I}(y)=\operatorname{Tr}\left(K_{[0, n+1]}^{*} P_{I} J_{0}\left(W_{0}\right) I_{[0, n+1]}^{r} y\right), y \in \mathfrak{A}_{[1, n]}
$$

Notice that $\left\{P_{I}: I \in\left\{1,2, \cdots, d^{\prime}\right\}^{n}\right\}$ is a family of minimal projections in $\mathfrak{A}_{[1, n]}$ such that $\sum P_{I}=1$. We denote by $\omega_{I}^{\prime}$ the positive linear functional on $\mathfrak{A}_{[0, n+1]}$ defined by the above relation for $y \in \mathfrak{A}_{[0, n+1]}$ :

$$
\omega_{I}^{\prime}(y)=\operatorname{Tr}\left(K_{[0, n+1]}^{*} P_{I} J_{0}\left(W_{0}\right) I_{[0, n+1]}^{*} y\right), y \in \mathfrak{A}_{[0, n+1]}
$$

Notice that $\omega_{I}^{\prime}\left|\mathfrak{A}_{[1, n]}=\omega_{I}\right| \mathfrak{A}_{[1, n]}$. We first express the state $\widehat{\omega}_{I}^{\prime}\left(=\omega_{I}^{\prime} / \omega_{I}^{\prime}(\mathbf{1})\right)$ as a finite convex combination of pure states on $\mathfrak{A}_{[0, n+1]}$ as follows : Consider $J_{0}\left(W_{0}\right)$ as a self-adjoint element in $J_{0}\left(M_{0}\right)$. Then $J_{0}\left(W_{0}\right)$ can be diagonalized as

$$
J_{0}\left(W_{0}\right)=\sum_{i=0}^{d^{\prime}} \lambda_{i} q_{i},
$$


where each $q_{i}$ is a minimal projection in $J_{0}\left(M_{0}\right)$. Thus as an element in $\mathfrak{A}_{[0, n+1]}, P_{I} J_{0}\left(W_{0}\right)$ can be written as

$$
P_{I} J_{0}\left(W_{0}\right)=\sum_{j=1}^{d^{\prime 2}} \tilde{\lambda}_{j} \tilde{p}_{j}
$$

where each $\tilde{p}_{j}$ is a minimal projection in $\mathfrak{A}_{\{0, n+1\}}$. For a given $j \in\left\{1,2, \cdots, d^{\prime 2}\right\}$, let $\widetilde{\omega}_{j}$ be the state on $\mathfrak{A}_{[0, n+1]}$ defined by

$$
\widetilde{\omega}_{j}(y)=\operatorname{Tr}\left(K_{[0, n+1]}^{*} \widetilde{p}_{j} K_{[0, n+1]} y\right) /\left(\operatorname{Tr} K_{[0, n+1]}^{*} \tilde{p}_{j} K_{[0, n+1]}\right)
$$

Then it follows that there exist $\beta_{j} \geq 0, j=1, \cdots, d^{2}$, such that $\sum \beta_{j}=1$ and

$$
\widehat{\omega}_{1}^{\prime}(y)=\sum_{j=1}^{d^{\prime 2}} \beta_{j} \widetilde{\omega}_{j}(y), y \in \mathfrak{A}_{[0, n+1]} .
$$

For any finite subset $\Lambda \subset \mathbf{Z}$, put $\mathfrak{H}_{\Lambda}=\left(\mathbf{C}^{d^{\prime}}\right)^{\Lambda}$. Notice that, if the state $\widehat{\omega}_{I}^{\prime}$ on $\mathfrak{A}_{[0, n+1]}$ is extended to $\mathfrak{L}\left(\tilde{\mathfrak{H}}_{[0, n+1}\right)$ by the means of the density matrix of $\widehat{\omega}_{I}^{\prime}$ in $\mathfrak{A}_{[0, n+1]}$, it follows that $S\left(\widehat{\omega}_{I} \mid \mathfrak{A}_{[0, n+1]}\right)=S\left(\widehat{\omega}_{I}^{\prime} \mid \mathfrak{L}\left(\mathfrak{A}_{[0, n+1]}\right)\right)$. Analogous equalities for $S\left(\widehat{\omega}_{I}^{\prime} \mid \mathfrak{A}_{[1, n]}\right)$ and $S\left(\widehat{\omega}_{I}^{\prime} \mid \mathfrak{A}_{\{0, n+1\}}\right)$ hold. Thus one can apply the triangle inecuality and the convexity relation [4, Proposition 6.2.25] for entropies to obtain

$$
S\left(\widehat{\omega}_{I} \mid \mathfrak{A}_{[1, n]}\right)=S\left(\widehat{\omega}_{I}^{\prime} \mid \mathfrak{A}_{[1, n]}\right) \leq S\left(\widehat{\omega}_{I}^{\prime} \mid \mathfrak{A}_{[0, n+1]}\right)+S\left(\widehat{\omega}_{I}^{\prime} \mid \mathfrak{A}_{\{0, n+1\}}\right)
$$

and

$$
S\left(\widehat{\omega}_{I}^{\prime} \mid \mathfrak{A}_{[0, n+1]} \leq \sum_{j=1}^{d^{\prime 2}} \beta_{j} S\left(\widetilde{\omega}_{j}\right)+\sum_{j=1}^{d^{\prime 2}} \eta\left(\beta_{j}\right)\right.
$$

respectively. Since $\tilde{p}_{j}$ is minimal in $2_{[0 . n+1]}$, it follows from Lemma 4.2 and $(4.11)$ that $\tilde{w}_{j}$ is a pure state on $\mathfrak{A}_{[0, n+1]}$ and so $S\left(\widetilde{\omega}_{j}\right)=0$ for all $j=1,2, \cdots, d^{\prime 2}$. Since $S\left(\widehat{\omega}_{I}^{\prime} \mid \mathfrak{A}_{\{0, n+1\}}\right) \leq$ $\log \left(\operatorname{dim}\left(\mathbf{C}^{2 d}\right)\right)$ and $\sum_{j=1}^{d^{\prime} 2} \eta\left(\beta_{j}\right) \leq \ln \left(d^{2}\right)$, we obtain from the above inequalities that

$$
S\left(\widehat{\omega}_{I} \mid \mathfrak{A}_{[1, n]}\right) \leq 4 \ln (d)
$$

This proves the proposition completely. 
Finally we turn to the proof of the theorem for the general case. Let $\left\{K_{1}, K_{2}, \cdots, K_{l}\right\}$ $\subset M_{0} \otimes M_{0}$ be the set of conditional density amplitudes and let the transition expectation $E$ be given by (2.12).

Proof of Theorem 3.2 for $l \geq 2$. We first construct a generalized quantum Markov $\operatorname{chain}(\widetilde{\mathfrak{L}}, \alpha, \widetilde{\omega})$ such that it is generated by a density matrix $\widetilde{W}_{0}$ and a single conditional density amplitude $\widetilde{K}$, and such that $(\mathfrak{A}, \alpha, \omega)$ is a sub-dynamical system of $(\widetilde{\mathfrak{A}}, \alpha, \widetilde{\omega})$, i.e., $\widetilde{\omega} \mid \mathfrak{A}=\omega$. We then apply the method employed in the proof for $l=1$. Let us enlarge the algebra $\mathfrak{A}$ as follows. Let $D_{0} \subset M_{l}(\mathbf{C})$ be the algebra of $l \times l$ diagonal matrices and let $\left\{e_{1}, e_{2}, \cdots, e_{l}\right\}$ be the minimal projections in $D_{0}$. Put

$$
\widetilde{M}_{0}=M_{0} @ D_{0}
$$

Denote by $\widetilde{\mathfrak{A}}$ the $C^{*}$-algebra generated by the one site algebra $\widetilde{M}_{0}$. For given density matrix $W_{0} \in M_{0}$ and $\left\{K_{1}, K_{2}, \cdots, K_{l}\right\} \subset M_{0} \odot M_{0}$, we write that

$$
\begin{aligned}
& \widetilde{W}_{0}=l^{-1} W_{0} \odot \mathbf{1}_{D_{0}} \quad\left(\in \widetilde{M}_{0}\right) \\
& \tilde{\Pi}=l^{-1 / 2} \sum_{j=1}^{l} \tilde{K}_{j} \oslash\left(e_{j} \bigcirc \mathbf{1}_{D_{0}}\right) \quad\left(\in \widetilde{M}_{0} \odot \widetilde{M}_{0}\right) .
\end{aligned}
$$

It is easy to check that $\widetilde{\omega} \mid \mathfrak{A}=\omega$, and $\widetilde{W}_{0}$ and $\widetilde{\Lambda^{-}}$satisfy the condition analogous to that of (2.6). Let $(\widetilde{\mathfrak{A}}, \alpha, \widetilde{\omega})$ be the generalized quant um Markov chain constructed from the pair $\left(\widetilde{W}_{0}, \widetilde{K}\right)$. For any subalgebra $\mathfrak{N} \subset \widetilde{\mathfrak{A}}$, let $\gamma: \mathfrak{N} \rightarrow \mathfrak{A}$ be the embedding map. Then the following inequality

$$
\begin{aligned}
& H_{\omega}\left(\mathfrak{L}_{[1, n]}, \mathfrak{A}_{[n+1,2 n]}, \cdots, \mathfrak{A}_{[n(k-1)+1, n k]}\right) \\
& \quad \geq H_{\tilde{\omega}}\left(\gamma\left(\mathfrak{A}_{[1, n]}\right), \gamma\left(\mathfrak{A}_{[n+1,2 n]}\right), \cdots, \gamma\left(\mathfrak{A}_{[n(k-1)+1, n k]}\right)\right)
\end{aligned}
$$

holds $[5,10]$.

We introduce a decomposition of the state $\tilde{\omega}$ similar to that given in (4.4)-(4.6). For fixed $k \geq 1$ and $n \geq 1$, let $\tilde{\mathcal{I}}$ be the multi-indices given by

$$
\widetilde{\mathcal{I}}=\left\{\widetilde{J}=\left(J_{1}, J_{2}, \cdots, J_{k}\right): J_{l} \in\left(\left\{1,2, \cdots, d^{\prime}\right\} \times\{1,2, \cdots, l\}\right)^{n}\right\}
$$

For each $J_{l}=\left(\left(i_{1}, j_{1}\right), \cdots,\left(i_{n}, j_{n}\right)\right) \in\left(\left\{1,2, \cdots, d^{\prime}\right\} \times\{1,2, \cdots, l\}\right)^{n}$, put

$$
\widetilde{P}_{J_{l}}=J_{n(l-1)+1}\left(P_{i_{1}} \odot \epsilon_{j_{1}}\right) \cdots J_{n l}\left(P_{i_{n}} \odot \epsilon_{j_{n}}\right)
$$


and for given $\tilde{J}=\left(J_{1}, \cdots, J_{n}\right)$ we write

$$
\widetilde{P}_{\tilde{J}}=\widetilde{P}_{J_{1}} \widetilde{P}_{J_{2}} \cdots \widetilde{P}_{J_{n}}
$$

for the index set $\tilde{\mathcal{I}}$, let

$$
\sum_{\tilde{j} \in \overline{\mathcal{I}}} \widetilde{\omega}_{\tilde{J}}=\widetilde{\omega}
$$

be the decomposition of the state $\widetilde{\omega}$ obtained by replacing $W_{0}, \pi$, and $J$ by $\widetilde{W}_{0}, \widetilde{K}$, and $\widetilde{J}$ respectively in (4.3)-(4.7). We then have the result analogous to Proposition 4.1 :

Proposition 4.3. There exists a constant $c$ independent of $n \in \mathbf{N}$ and $I \in\left(\left\{1,2, \cdots, l^{\prime}\right\} \times\right.$ $\{1,2, \cdots, l\})^{n}$ such that the bound

$$
S\left(\widehat{\tilde{\omega}}_{I} \mid \gamma\left(\mathfrak{A}_{[1, n]}\right)\right) \leq c
$$

holds uniformly in $n$ and $I$.

Now the proof of Theorem 3.2 for $l \geq 2$ follows from (4.16), Proposition 4.3 and the method used in the proof of Theorem 3.2 for $l=1$. We only note that $S\left(\widetilde{\omega} \mid \gamma\left(\mathfrak{A}_{[1, n]}\right)\right)=$ $S\left(\omega \mid \mathfrak{A}_{[1, n]}\right)$, and leave the detailed proof to the reader.

Proof of Proposition 4.3. Let $\widetilde{\omega}_{I}^{\prime}$ be the positive linear functional on $\widetilde{\mathfrak{A}}_{[0, n+1]}$ defined by a relation analogous to that in (4.10). As the relation in the above of (4.11), one may wite

$$
\widetilde{P}_{I} J_{0}\left(\widetilde{W}_{0}\right)=\sum_{j=1}^{\left(l d^{\prime}\right)^{2}} \widetilde{\lambda}_{j} \widetilde{q}_{j}
$$

where each $\widetilde{q}_{j}$ is a minimal projection in $\widetilde{\mathfrak{A}}_{[0, n+1]}$. Then as in (4.12) we have

$$
\widehat{\bar{\omega}}_{I}^{\prime}(y)=\sum_{j=1}^{\left(l d^{\prime}\right)^{2}} \beta_{j} \widetilde{\omega}_{j}(y), \quad y \in \widetilde{\mathfrak{A}}_{[0, n+1]},
$$

where each $\widetilde{\omega}_{j}$ is a pure state on $\widetilde{\mathfrak{A}}_{[0, n+1]}$. 
Next, one may use the argument similar to that used in the proof of (4.13) to derive the inequality

$$
S\left(\widehat{\tilde{\omega}}_{I} \mid \gamma\left(\mathfrak{A}_{[1, n]}\right)\right) \leq S\left(\hat{\tilde{\omega}}_{I}^{\prime} \mid \gamma\left(\mathfrak{A}_{[0, n+1]}\right)\right)+S\left(\hat{\tilde{\omega}}_{I}^{\prime} \mid \gamma\left(\mathfrak{A}_{\{0, n+1\}}\right)\right)
$$

That is, extend the state $\widehat{\hat{\omega}}_{I}^{\prime} \mid \gamma\left(\mathfrak{A}_{[0, n+1]}\right)$ to $\mathcal{L}\left(\mathfrak{\mathfrak { H }}_{[0, n+1]}\right)$ by the density matrix of $\widehat{\tilde{\omega}}_{I}^{\prime}$, and then use the triangle inequality for entropies. Notice that

$$
\widetilde{\mathfrak{A}}_{[0, n+1]}=\mathfrak{A}_{[0, n+1]} \otimes D_{[0, n+1]},
$$

where $D_{I}=\otimes_{n \in I} J_{n}\left(D_{0}\right)$ is an abelian algebra for each $I \subset Z$. Since each state $\widetilde{\omega}_{j}$ in (4.22) is pure, there exist pure states $\rho_{j, 1}$ and $\rho_{j, 2}$ on $\mathfrak{A}_{[0, n+1]}$ and $D_{[0, n+1]}$ respectively such that

$$
\widetilde{\omega}_{j}=\rho_{j, 1} \odot \rho_{j, 2} \text {. }
$$

Thus $S\left(\widetilde{\omega}_{j} \mid \gamma\left(\mathfrak{A}_{[0, n+1]}\right)\right)=S\left(\rho_{j, 1}\right)=0$, and so the proposition follows from the argument similar to that used in the below of (4.13). This completes the proof of Proposition 4.3.

Acknowledgement. The author would like to thank Prof. Størmer for his warm hospitality when the author visited the University of Oslo and for helpful discussions which enable to improve the manuscript considerably.

\section{References}

[1] Accardi, L. and Frigerio, A., Markov cocycles, Proc. R. Ir. Acad. 83 A, 251-269 (1983).

[2] Accardi, L. and Watson, G.S., Quantum random walks, Lecture Notes in Math. 1936, 73-78, Springer-Verlag 1989.

[3] Besson, O., On the entropy of quantum Markov states, Lecture Notes in Math. 1136, 81-89, Springer-Verlag 1985.

[4] Bratteli, O. and Robinson, D.W., Operator algebras and quantum statistical mechanics. I, II, Springer-Verlag 1981.

[5] Connes, A., Namhofer, H. and Thirring W., Dynamical entropy of $C^{*}$-algebras and von Neumann algebras. Commun. Math. Phys. 112, 691-719 (1987). 
[6] Connes, A. and Størmer, E., Entropy of automorphisms of $I I_{1}$ von Neumann algebras. Acta math. 143, 289-306 (1975).

[7] . Kolmogorov, A.N., Dokl. Akad. Nauk. 119, 861 (1958).

[8] Narnhofer, H. and Thirring, W., Dynamical entropy of quasi-free automorphisms. Letters Math. Phys. 14, 89-96 (1987).

[9] Narnhofer, H. and Thirring, W., Dynamical entropy of quantum systems and their Abelian counterpart. To appear.

[10] Ohya, M. and Petz, D., Quantum entropy and its use, Springer-Verlag 1993.

[11] Park, Y.M. and Shin, H.H., Dynamical entropy of quasi-local algebras in quantum statistical mechanics. Commun. Math. Phys. 144, 149-161 (1992).

[12] Park, Y.M. and Shin, H.H., Dynamical entropy of space translations of CAR and CCR algebras with respect to quasi-free states. Commun. Math. Phys. 152, 497-537 (1993).

[13] Quasthoff, U., Shift automorphisms of the hyperfinite factor, Math. Nachrichten 131, 101-106 (1987).

[14] Sinai, Ya. G., Dokl. Akad. Nauk., 768 (1959).

[15] Størmer, E., Entropy of some automorphisms of the $I_{1}$-factor of the free group in infinite number of generators. Invent. Math. Phys. 110, 63-73 (1992).

[16] Størmer, E., Entropy in operator algebra, Preprint (1992).

[17] Størmer, E., and Voiculescu, D., Entropy of Bogoliubov automorphisms of the canonical anticommutation relations. Commun. Math. Phys. 133, 521-542 (1990).

[18] Walters, P., An introduction to ergodic theory. Graduate Texts in Math. 79, SpringerVerlag 1982. 
